\title{
La Ética de la Investigación en épocas de Pandemia
}

\section{Research ethics in the pandemic era}

La pandemia por Covid-19 constituye una situación excepcional que impondrá nuevos desafíos y una acuciante necesidad de encontrar nuevas soluciones. Uno de esos desafíos será llevar adelante diversos tipos de investigaciones biomédicas en un contexto de enorme vulnerabilidad, tanto de nuestra población como del sistema de salud que deberá darles respuesta.

Habrá un enorme desequilibrio entre las necesidades médicas y la disponibilidad de recursos sanitarios. El mayor desafío será cómo asignar de manera justa los recursos sanitarios, privilegiando "la mayor esperanza de vida", en un marco ético que contemple principios claves como la equidad en el acceso, la responsabilidad. precaución y proporcionalidad terapéutica, en un marco de respeto a los derechos, la seguridad, el bienestar, la dignidad y la intimidad de las personas.

Otro será el de tomar decisiones científicamente fundadas sobre la escasa información disponible acerca de cómo tratar esta nueva enfermedad y el uso compasivo de medicamentos con indicaciones "Off Label”.

Nunca como ahora, será médica y éticamente, tan necesario recurrir a la investigación médica.

Cabe aclarar que No toda recolección sistemática de datos constituye investigación con seres humanos. La recopilación de datos epidemiológicos o el envió de información a bases de datos oficiales No constituyen Investigación en Seres Humanos sino herramientas básicas para la toma de decisiones de Salud Pública. No requieren de autorizaciones especiales y no se encuentran bajo la órbita de los comités de ética de la investigación.

La investigación patrocinada deberá enfocarse en la eficacia y seguridad de nuevos métodos diagnósticos, de prevención y de tratamiento de este problema de salud. Esta investigación será crucial para reducir la incertidumbre acerca de la pandemia y sus consecuencias. Tenemos el deber ético de hacer investigación durante el brote para comprender la enfermedad y para evaluar la seguridad y la eficacia de todas las propuestas de exámenes de diagnóstico, tratamientos, vacunas y estrategias de manejo. Es importante que los estudios aborden las necesidades y las prioridades locales y que su diseño sea acorde a las capacidades locales y aceptable para la población donde se llevará a cabo.

Revista Methodo: Investigación Aplicada a las Ciencias Biológicas. Facultad de Medicina. Universidad Católica de Córdoba. Jacinto Ríos 571 Bo Gral. Paz. X5004FXS. Córdoba. Argentina. Tel.: (54) 3514517299 / Correo: methodo@ucc.edu.ar / Web: methodo.ucc.edu.ar | EDITORIAL Rev. Methodo 2020;5(2):38-40. 
Sin embargo, investigar en este contexto acarrea muchos riesgos, más allá de los inherentes a la propia condición humana y a la enfermedad que enfrentamos. Todos los estándares serán indispensables, pero no suficientes para asegurar la eticidad de las investigaciones en tiempos de pandemia. A pesar de ello, una negativa a realizarla conduciría a la "parálisis de la medicina" en detrimento de nuestro pueblo.

Tanto la Administración Nacional de Medicamentos, Alimentos y Tecnología Médica (ANMAT) como en Consejo de Evaluación de Investigaciones en Salud (CoEIS) han dado prioridad a investigaciones sobre COVID-19 y han hecho recomendaciones para la mitigación del riesgo para personas actualmente en investigación.

Los comités de ética de la investigación en salud humana (Como el CIEIS-Fabiola), jugarán un papel central en esta contingencia, contribuyendo a la calidad científica y consistencia metodológica en la ejecución investigaciones biomédicas y asegurando la defensa de la integridad, la dignidad y los derechos humanos de las personas, no sólo en lo referido a los riesgos asumidos por su participación en los estudios, sino por el aprovechamiento compartido de los beneficios.

En épocas de pandemia, no serán suficientes los clásicos elementos del enfoque principialista, Autonomía, Beneficencia, Justicia y No Maleficencia. Será necesario recurrir a los principios cautelares, como el de responsabilidad (Hans Jonas), de Precaución, Protección (Kottow), y a otros como el de Intervención (Garrafa), o los de dignidad, Bioética y derechos humanos (Tealdi y otros), así como al de vulnerabilidad y justicia social, entre otros, para asegurar que la pandemia no imponga una carga desproporcionada sobre ningún grupo, en especial los más pobres y desfavorecidos.

Finalmente, será necesario realizar revisiones éticas aceleradas durante la pandemia. La investigación con seres humanos durante emergencias debe contar con garantías éticas mayores, no menores que en situaciones ordinarias. Esto implica acelerar los procesos de evaluación, pero no a expensas de perder rigurosidad en la revisión ética, tanto en los aspectos científicos y metodológicos como en el proceso de información y consentimiento informado, la obtención y procesamiento de muestras y las garantías de tratamiento al final de los estudios, que serán exigidos, aun en la emergencia, como así también en la necesidad de compartir inmediatamente los resultados de la investigación, para el bien de todos.

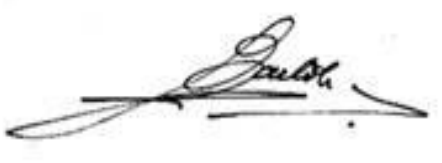

Prof. Julio Bartoli Coordinador CIEIS-Fabiola

\section{Referencias}

Orientación ética sobre cuestiones planteadas por la pandemia del nuevo coronavirus (COVID-19). Washington, D.C., 16 de marzo del 2020. OPS-OMS. Disponible en:

https://www.paho.org/es/documentos/orientacion-etica-sobre-cuestiones-planteadas-por-pandemia-nuevo-coronavirus-covid-19. World Health Organization. Ethics in epidemics, emergencies and disasters: Research, surveillance and patient care. Training manual. Ginebra: Organización Mundial de la Salud; 2015. Disponible en:

Revista Methodo: Investigación Aplicada a las Ciencias Biológicas. Facultad de Medicina. Universidad Católica de Córdoba. Jacinto Ríos 571 Bo Gral. Paz. X5004FXS. Córdoba. Argentina. Tel.: (54) 3514517299 / Correo: methodo@ucc.edu.ar / Web: methodo.ucc.edu.ar | EDITORIAL Rev. Methodo 2020;5(2):38-40 
Bartoli J. La Ética de la Investigación en épocas de Pandemia.

http://apps.who.int/iris/bitstream/10665/196326/1/9789241549349 eng.pdf.

Organización Mundial de la Salud. Investigaciones para una cobertura sanitaria universal. Informe sobre la salud en el mundo 2013. Ginebra: OMS, 2013. Disponible en:

http://apps.who.int/iris/bitstream/10665/85763/1/9789240691223_spa.pdf?ua=1

Millum J, Beecroft B, Hardcastle TC, et al. Emergency care research ethics in low-income and middle-income countries. BMJ Global Health 2019;4: e001260.

Global Forum on Bioethics in Research (GFBR). Meeting report: Emerging epidemic infections and experimental medical treatments. Annecy, Francia. 3 y 4 de noviembre del 2015. Disponible en:

http://www.gfbr.global/wp-content/uploads/2016/03/GFBR-2015-meeting-report-emergingepidemic-infections-and-experimentalmedical-treatments.pdf.

Consejo de Organizaciones Internacionales de las Ciencias Médicas (CIOMS). Pautas éticas internacionales para la investigación relacionada con la salud con seres humanos, Cuarta Edición. OPS y CIOMS, 2016. Disponible en:

https://iris.paho.org/bitstream/handle/10665.2/34457/9789290360902-spa.pdf? sequence=5\&isAllowed=y.

\section{(c) (1) (8) \\ BY NC SA}

Revista Methodo: Investigación Aplicada a las Ciencias Biológicas. Facultad de Medicina. Universidad Católica de Córdoba. Jacinto Ríos 571 Bo Gral. Paz. X5004FXS. Córdoba. Argentina. Tel.: (54) 3514517299 / Correo: methodo@ucc.edu.ar / Web: methodo.ucc.edu.ar | EDITORIAL Rev. Methodo 2020;5(2):38-40 